\title{
Experimental investigation of supercavitating flows
}

\author{
Byoung-Kwon Ahn, Tae-Kwon Lee, Hyoung-Tae Kim and Chang-Sup Lee \\ Department of Naval Architecture and Ocean Engineering, College of Engineering, \\ Chungnam National University, Korea
}

\begin{abstract}
When the object is traveling in the water at tremendously high speeds, the cavity forms and grows up at a fore part of the object called cavitator, and the object is eventually enveloped by vaporized water, supercavitation. As a result, the only part of the object in direct contact with the water is the cavitator, so skin-friction drag is significantly reduced. This is why recently supercavitating objects have been interested in many applicable fields. In this study we are focused out attention on supercavitating flows around various shapes of two and three dimensional cavitators. First, general features of supercavitation are examined by analyzing results obtained by the previously developed numerical method. Second, experimental observations are carried out at a cavitation tunnel at the Chungnam National University (CNU CT), and supercavity dimensions are scrutinized.
\end{abstract}

KEY WORDS: Cavitator; Cavitation; Super-cavitation; Cavitation tunnel; Supercavity length; Supercavity width.

\section{INTRODUCTION}

Cavitation occurs if the pressure declines to some point below the saturated vapor pressure of the liquid. When a volume of liquid is subjected to a sufficiently low pressure, it may rupture and form a cavity and it is visible. This is the condition of cavitation inception. Cavitation may occur on the blade of a rapidly rotating propeller in the water or on any object vibrating in the liquid with sufficient amplitude and acceleration. In such devices, cavitation causes a great deal of noise and vibration, erosion to components and also a loss of efficiency of the system. Cavitation is, in many cases of marine related engineering, an undesirable phenomenon.

However, when a cavity grows enough at high speed to envelop the object, it is called supercavitation. If the submerged object in the water is encompassed by the supercavity, it is possible to gain tremendously high speed by reducing skin-friction drag in the water, and it has been attempted to use for military purposes such as projectiles or torpedoes. In principle it could be extended to include underwater vehicles. A supercavitating object has a special shaped nose called cavitator, which is generally flat with sharp edges. When the object is moving in the water at high speeds, the cavitator disperses the water outward at speeds. There is a tremendous drop in pressure in where the water passes over the sharp edge of the periphery of the cavitator. Then the cavity is initially formed at the cavitator, and it persists traveling with the object, closing in behind of it. There are two ways to achieve the supercavity: by increasing object's speed enough that is called natural supercavitation, or by decreasing the ambient pressure using internally generated ventilated gas around the object that is artificial or ventilated supercavitation.

The studies on supercavitating flows have been started in the late 1940s after Reichardt's pioneering work. Reichardt (1946) showed the case for ventilated supercavity behind disks, and Self and Ripken (1955) investigated steady-state cavity in a free-jet water tunnel. Tulin (1953) presented the two-dimensional slender body theory for modeling supercavitating flows, and Gilbarg (1957) showed a solution of non-thin wedges by applying hodograph method. Waid (1957) measured cavity shapes for a flat plate and wedges. After these remarkable studies, related works have not been presented for a long time until the end of 1990s.

Corresponding author: Hyoung-Tae Kim

e-mail:h-tkim@cnu.ac.kr 
During this period partial cavity problems on hydrofoils and marine propellers were mainly interested. Semenenko (2001) suggested calculation methods of the ventilated supercavity. He derived approximated equations of the axially symmetric supercavity with using the theorem of momentum and asymptotic theory of slender body. Alyanak, Venkayya, Grandhi and Penmetsa (2004) showed algorithm to determine the optimal configuration of the supercavitating body. Recently, numerical modeling and techniques for two or multi-phase flow analysis have been developed by Kunz, Lindau, Billet and stinegring (2001) and Saurel and Le Metayer (2001).

First, supercavity shape such as length and maximum width was the most important feature of practical concern for the supercavitating object. So previous studies mainly devoted to measure or calculate the cavity shape at relatively low cavitation numbers. However, it is also important to understand the growth phase of the supercavity at high cavitation numbers as well because, during this period, physically and technically significant circumstances occur.

In the present study, we were focused our attention on experimental observations of the early stage to create the supercavitation on several shapes of two and axisymmetric cavitators. In order to determine the cavity shape and characterize supercavitating events in more detail, high-speed camera was used and results were carefully examined.

\section{PREVIOUS RESULTS: GENERAL FEATURES OF SUPERCAVITATION}

The main parameters that affect the inception and subsequent character of cavitation and supercavitation are absolute pressure, velocity, and vapor pressure. The relevant non-dimensional parameter describing the occurrence of cavitation and the details of the resulting flow is the cavitation number:

$$
\sigma \equiv \frac{p_{\infty}-p_{v}}{1 / 2 \rho V_{\infty}^{2}}
$$

where $p_{\infty}$ is the pressure of undisturbed liquid, $p_{v}$ is the vapor pressure of liquid, $\rho$ is the density of liquid, and $V$ is the velocity of undisturbed liquid relative to the body. The value of $\sigma$ at which cavitation inception appears is designated as $\sigma_{i}$ and a theoretical value of $\sigma_{i}$ is the magnitude of the pressure coefficient $\left(-C_{p}\right)$ given by

$$
-C_{P}=\frac{p_{\infty}-p}{1 / 2 \rho U_{\infty}^{2}}>\frac{p_{\infty}-p_{v}}{1 / 2 \rho U_{\infty}^{2}} \equiv \sigma
$$

Thus cavitation inception means the appearance of cavities at or near the place on the object where the minimum pressure is acted. As the velocity is increased (cavitation number is decreased), the pressure at other points along the surface of the body may drop to the vapor pressure. Then the cavity spreads from the location of the inception point to the wake. As the cavitation

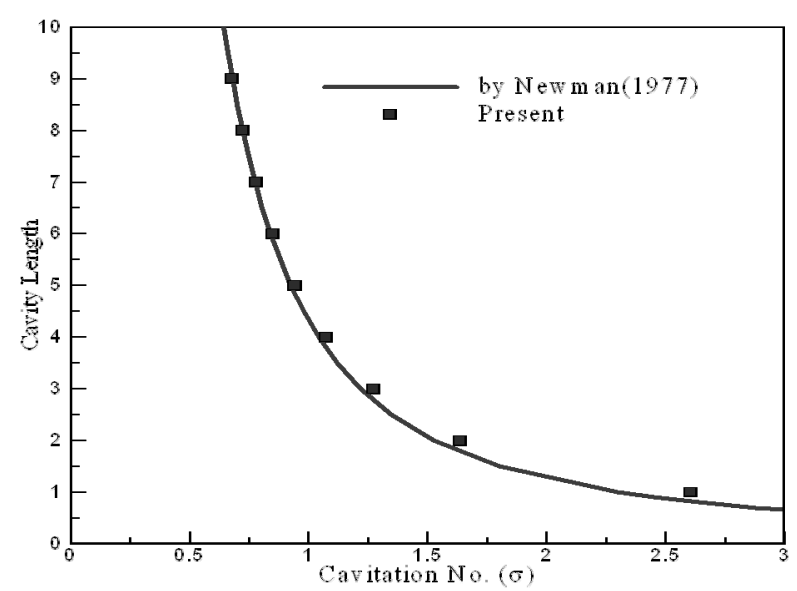

Fig. 1 Cavity length versus cavitation number for two-dimensional wedge shape cavitator.

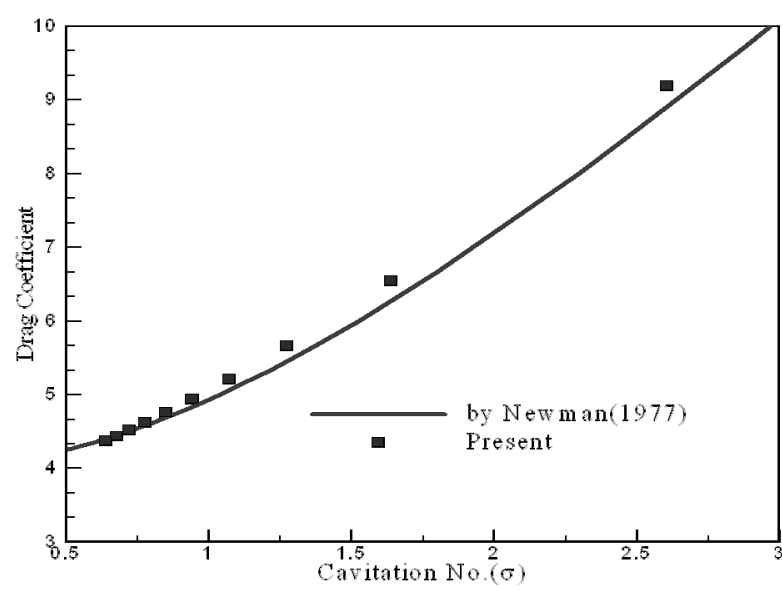

Fig. 2 Drag coefficient versus cavitation number for two-dimensional wedge shape cavitator. 
number is reduced further, the cavity grows up and wide-spreads, and finally it becomes supercavity. In our previous work (Ahn, Lee and kim, 2010), we showed general features of supercavitation generated by symmetrical wedge type cavitator; for decreasing values of the cavitation number, the cavity length increases while the drag force is reduced. We developed a numerical method which is based on inviscid flow and evaluated supercavitation yielding a sufficiently large enough cavity to envelop the body. Fig. 1 and Fig. 2 show predicted cavity length and drag coefficient comparing with the analytic solution which is derived on the basis of the linear theory by Newman (1977). This analytic solution is invalid if the cavity length is substantially shorter than the cavitator length, or if the cavitation number is too large. But this analysis is enough to show the main feature of the supercavity.

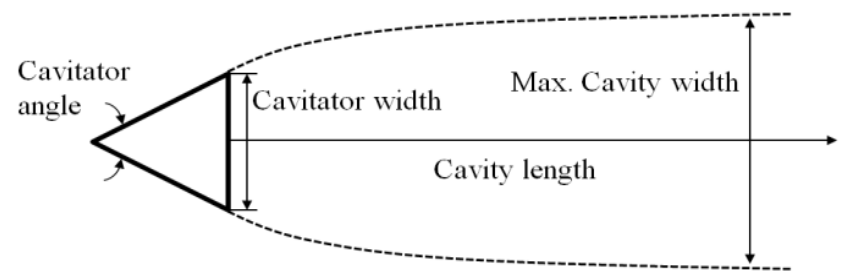

Fig. 3 Configuration of two-dimensional cavitator.

In addition, we, here, applied previously developed numerical method to prediction of cavity length, maximum width and drag forces for wedge shape cavitator with different angles (see Fig. 3). Supercavity configuration and drag are the features of

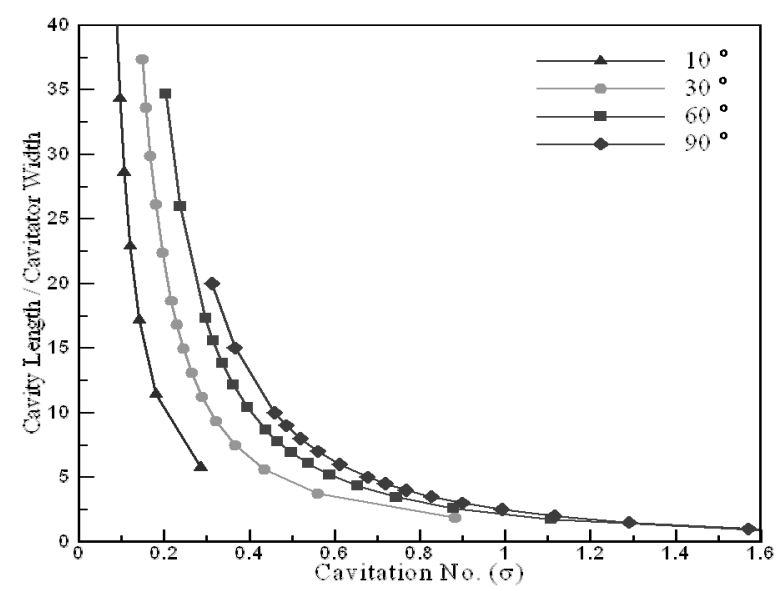

Fig. 4 Predicted cavity length for different cavitator angles.

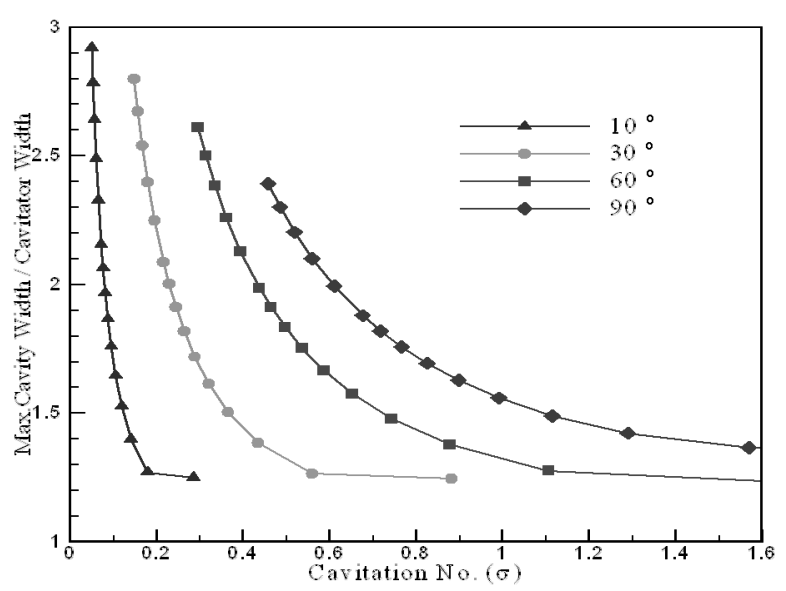

Fig. 5 Predicted maximum cavity width for different cavitator angles.

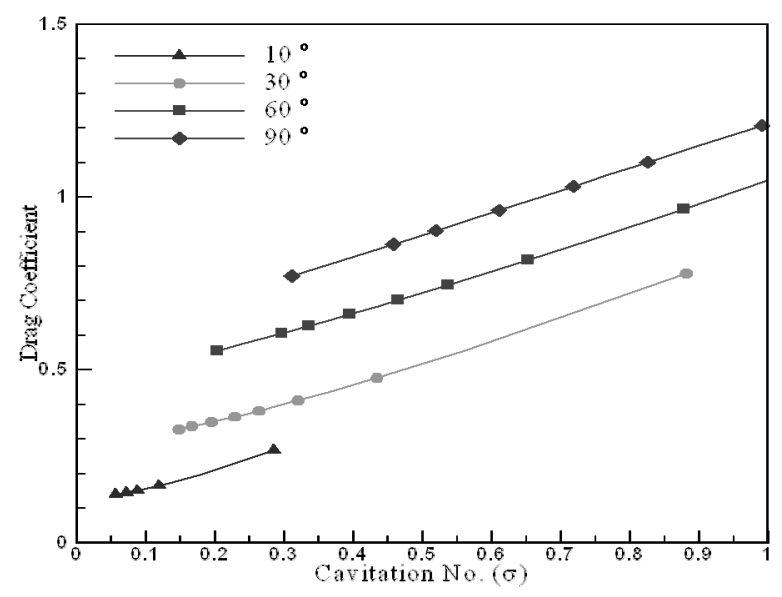

Fig. 6 Predicted drag coefficient for different cavitator angles. 
practical concern for symmetrical cavities. The longer and wider cavity, but the smaller drag is the better. As shown in Fig. 4 and Fig. 5, blunt cavitator generates longer length and wider width rather than the slender cavitator. In these cases, the cavity shape is nearly ellipsoidal and the drag variation with cavitation number is nearly linear as shown in Fig. 6.

\section{EXPERIMENTAL APPRATUS AND SETUP}

Main object of this work is to scrutinize and take a close examination of the supercavity. We specially focused our attention on investigation of the early stage of supercavitation; how the cavity occurs, and grows, how the wake field of the cavity pulsates, where how reentrant jet does act. Historically the downstream of the supercavity was one of concerns, so many previous researches have been devoted to model for a finite wake flow behind the cavitator. Supercavity has the characteristics of classical free streamline flows. The cavity inside is strictly at constant pressure, and interfaces of the cavity and the liquid are essentially free-stream surfaces of constant velocity. However, it is complex to describe the unknown freestream surface properly, and there have been many theoretical models for finite wake and cavity closure. Knapp, Daily and Hammitt (1979) reviewed well about these issues. In order to clear of these interests we recorded the growth phase of the supercavity using 250,000 fps high-speed camera (in more detail, see Table 1) and examined the data comparing with that of general video camera. Experimental observations were carried out at the cavitation tunnel of the Department of Naval Architecture and Ocean Engineering, Chungnam National University. Tunnel specification is shown in Table 2.

Table 1 Specification of the high-speed camera.

\begin{tabular}{|c|c|}
\hline Model name & Photron ultima APX-RS \\
\hline Max Frame Rate & $250,000 \mathrm{fps}$ \\
\hline Max Resolution & $1,024 \times 1,024$ \\
\hline Max at Max Res. & $3,000 \mathrm{fps}$ \\
\hline Max Record Time & $12.3 \mathrm{sec}$ \\
\hline Max Shutter Speed & 2 us \\
\hline Sensor Type & CMOS \\
\hline Lens Mount & F \& C-Mount \\
\hline Interface Options & Firewire, Optical, GigE \\
\hline Lamp & $12,500 \operatorname{lm}(150 \mathrm{~W})$ \\
\hline
\end{tabular}

Table 2 Specification of the cavitation tunnel.

\begin{tabular}{|c|c|c|c|}
\hline Tunnel $(L \times H)$ & $6.8 \mathrm{~m} \times 2.0 \mathrm{~m}$ & Speed (max) & $15 \mathrm{~m} / \mathrm{s}$ \\
\hline Test section $(D \times B)$ & $120 \mathrm{~mm} \times 50 \mathrm{~mm}$ & Cav. No. Range & $0.4 \sim 5.0$ \\
\hline Pressure $(\mathrm{min})$ & $10 \mathrm{kPa}$ & Pressure (max) & $300 \mathrm{kPa}$ \\
\hline
\end{tabular}

\section{TOW-DIMENSIONAL SUPERCAVITATION}

We observed supercavity profiles following wedge angle $30^{\circ}, 45^{\circ}$, and flat plates. We measured the effects of the body placed behind the wedge and flat plate. We recorded the cavity growth using $30 \mathrm{fps}$ general video camera and 250,000 fps highspeed camera, and compared results together. In this try, 50,000 fps image was sufficient to find a process of cavity growth in more detail. As described above, cavity length and maximum width are important features of practical concern for supercavitating object. We found that one may yield big differences between the results from a general camcorder and high-speed camera, which were determined by measuring averaged instantaneous images. The results are compared in Fig. 7; cavity lengths are overestimated by using general camcorder films, and the difference is increased as the cavitation number is reduced. This feature can be identified by comparing successive events of the supercavity recorded by general camcorder and high-speed camera as shown in Fig. 10. 


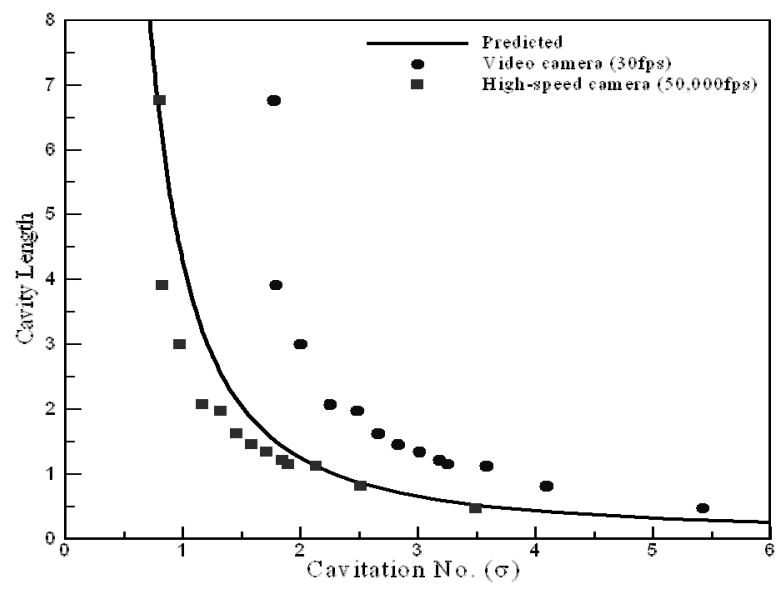

Fig. 7 Comparison of cavity length recorded by a $30 \mathrm{fps}$ general camcorder and 50,000 fps high-speed camera images.

Cavity shapes for a $30^{\circ}$ and $45^{\circ}$ wedge and flat plate cavitator and also same cavitators with a body which is 0.7 times thick and 5 times long of the cavitator width. In the experiments the ratio of cavitator thickness $(0.02 \mathrm{~m})$ to tunnel height $(0.12 \mathrm{~m})$ was 0.17. In Fig. 8 and Fig. 9, cavity length and width generated by different two-dimensional cavitators are compared respectively. The results follow expected trends; the flat plate cavitator generates longer and wider cavities rather than that of wedge type cavitators. In all cases with the body behind the cavitator, cavity growths are delayed. One may understand that detachment of the cavity occurs close to the point where the boundary pressure falls to the vapor pressure. The detachment position is affected by the cavitator size because the effect of surface tension is larger in small cavities where interface curvatures are higher. The initial slope of the cavity is continuous with the slope of the body surface. These features were captured by the high-speed camera records. In Fig. 10, high-speed filim strips of cavitation in the wake of a $45^{\circ}$ cavitator are compared with that of $30 \mathrm{fps}$ video camcorder. Since wakes are liquid-filled and not vaporous fluid, zones of high shear develop between the fast moving flow and slow circulating wake flow. As a result, high-intensity separating vortices are formed in the wake behind the cavitator. As the cavitation number is decreased, on the other hand, as the flow speed is increased, finite-length cavity is formed. However, vortices are still remaining behind the cavity until the cavity grows enough to form an ellipse. This sequence of events is not visible using general video camera records. As the cavitation number is reduced to about 0.83 , strictly limited to this case, the reentrant flow falls into the cavity interfaces, and it is removed by the high speed flow. These Features are important because that the pulsating and shedding vortices behind the early-stage-cavity cause the supercavitating object to be forced unsteady, which should be controlled unerringly to maintain its designated route. Through the tests, it was confirmed that one may overestimate supercavity configurations and may not capture hidden inherent-characteristics of the cavity using a low speed film (general video camera).

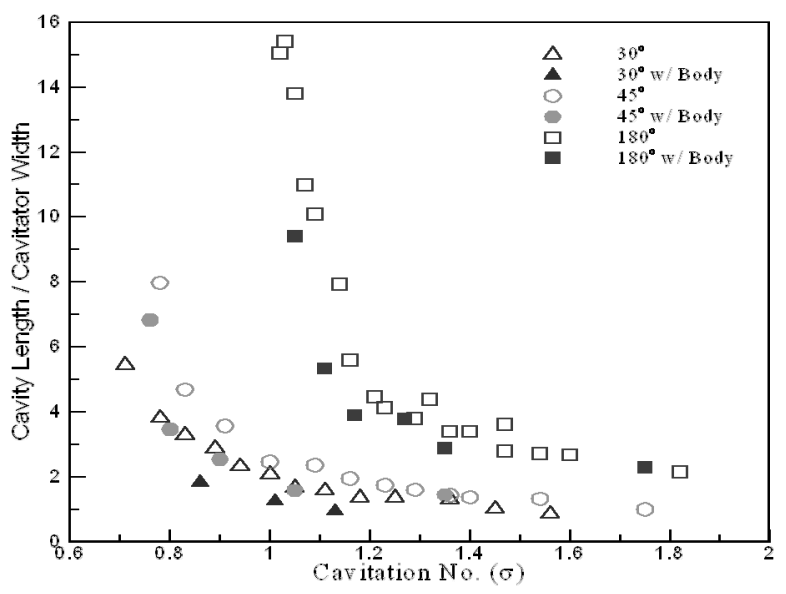

Fig. 8 Non-dimensional cavity length versus cavitation number for different two-dimensional cavitators.

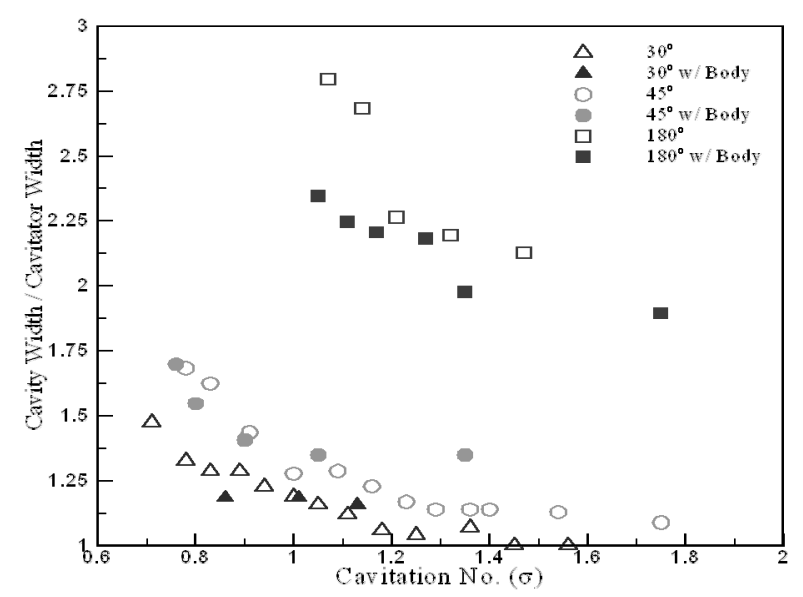

Fig. 9 Non-dimensional cavity width versus cavitation number for different two-dimensional cavitators. 


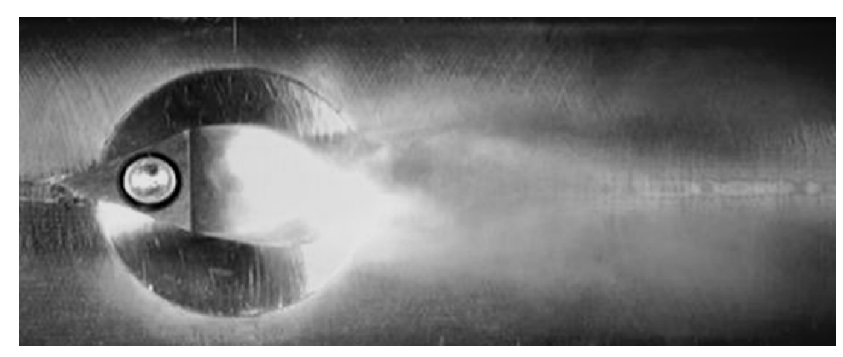

$\sigma=1.40$

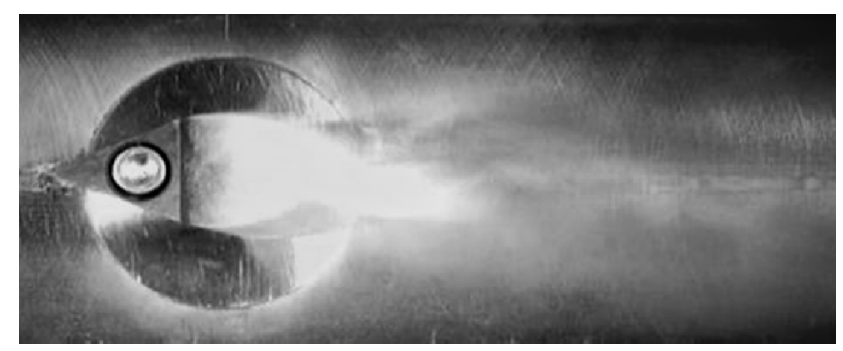

$\sigma=1.23$

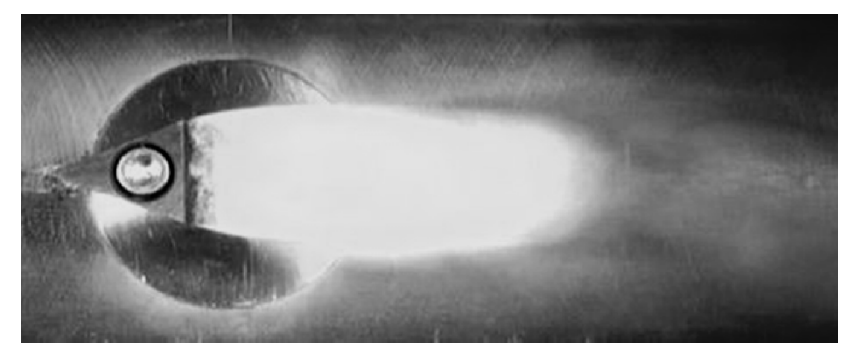

$\sigma=0.91$

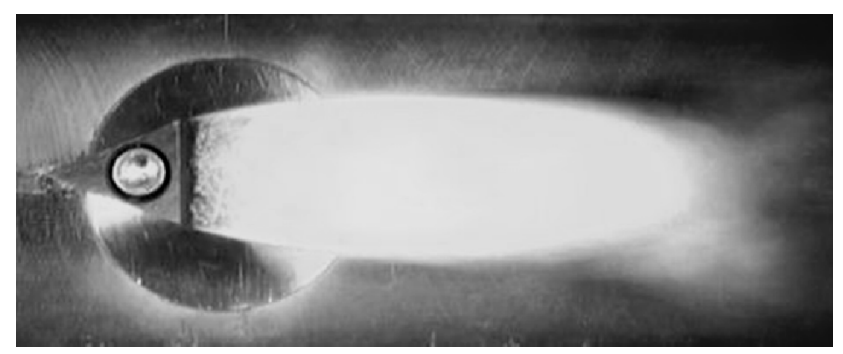

$\sigma=0.83$

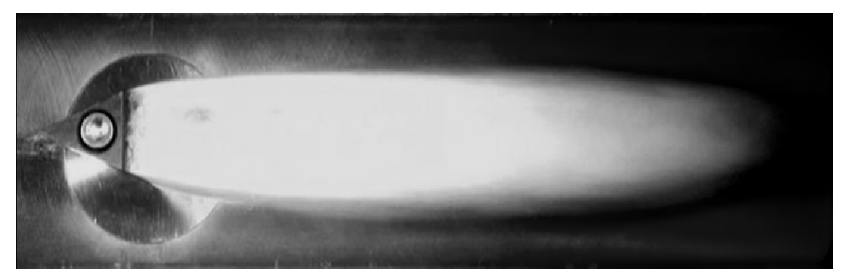

$\sigma=0.78$

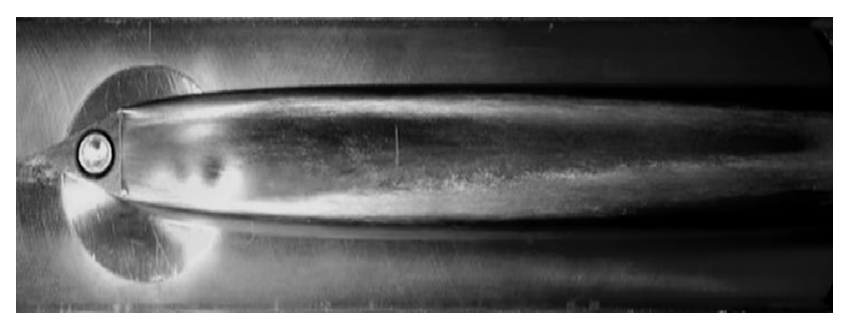

$\sigma=0.69$

(a) General video camera (30 fps) strips.

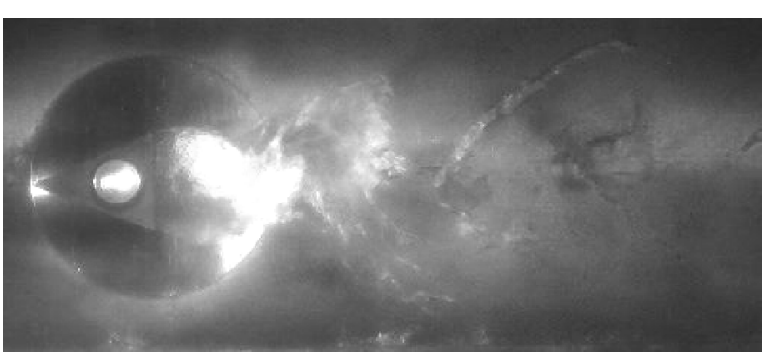

$\sigma=1.40$

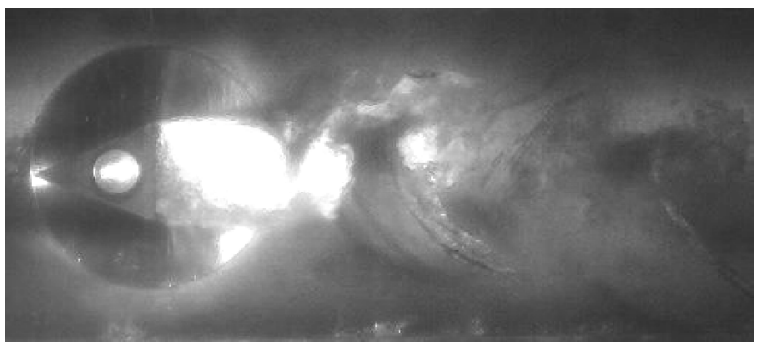

$\sigma=1.23$

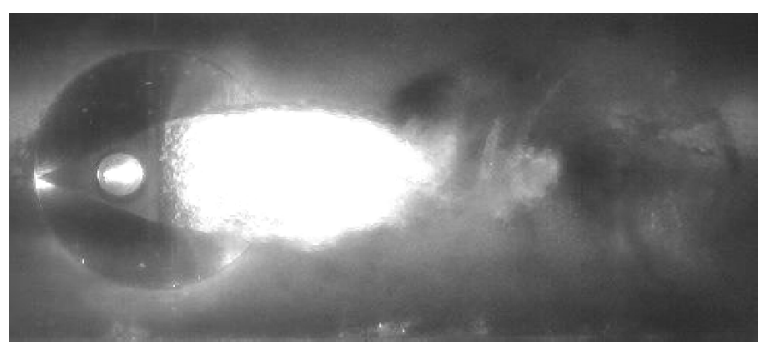

$\sigma=0.91$

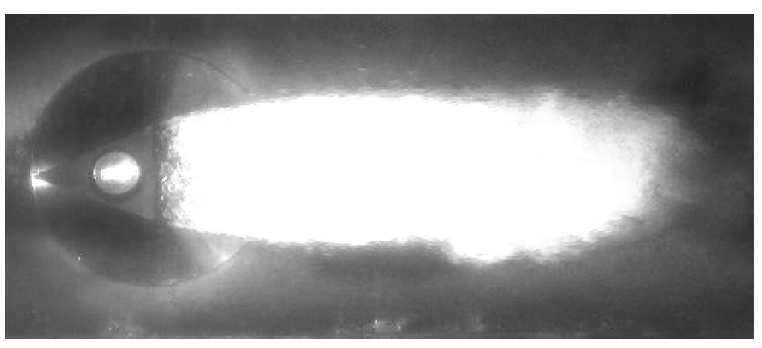

$\sigma=0.83$

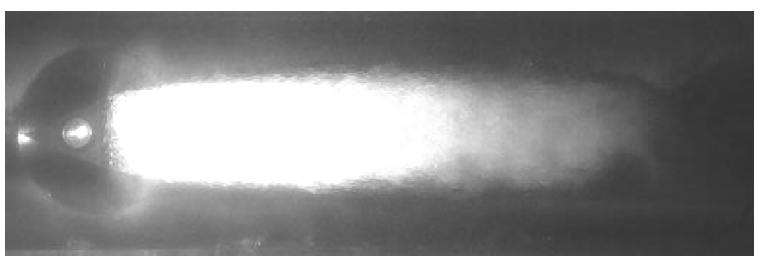

$\sigma=0.78$

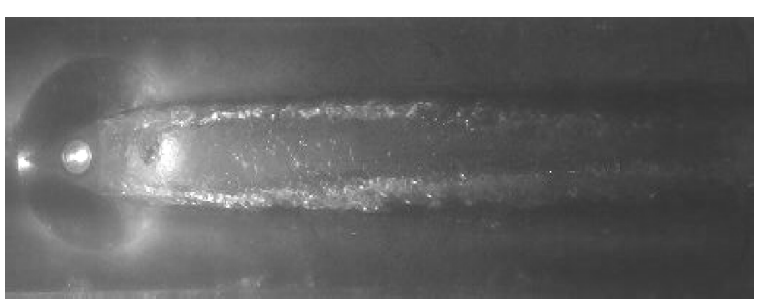

$\sigma=0.69$

(b) High-speed camera $(50,000 \mathrm{fps})$ strips.

Fig. 10 Sequence of events of the supercavity in the wake of a $45^{\circ}$ cavitator (flow speed $V=9.4 \mathrm{~m} / \mathrm{s}$ ). 


\section{AXISYMMETRIC SUPERCAVITATION}

We also observed supercavity profiles following three-dimensional cone and disk shape cavitators; first model has a $5 \mathrm{~mm}$ rounded tip, second one is $45^{\circ}$ cone, third one has a flat plate (disk), and the other has a $10 \mathrm{~mm}$ hole at the center of the disk. Specifications of test models are shown in Fig. 11. In practice to gain sufficient speed that is enough to generate the supercavity, supercavitating object generally uses special fuels such as metallic powders instead of existing gas or liquid fuels. Metallic powders are reactivated with seawater inhaled from the cavitator. So we observed the effects of a water intake using the last mode and compared supercavity features for both cases of with and without water intake.

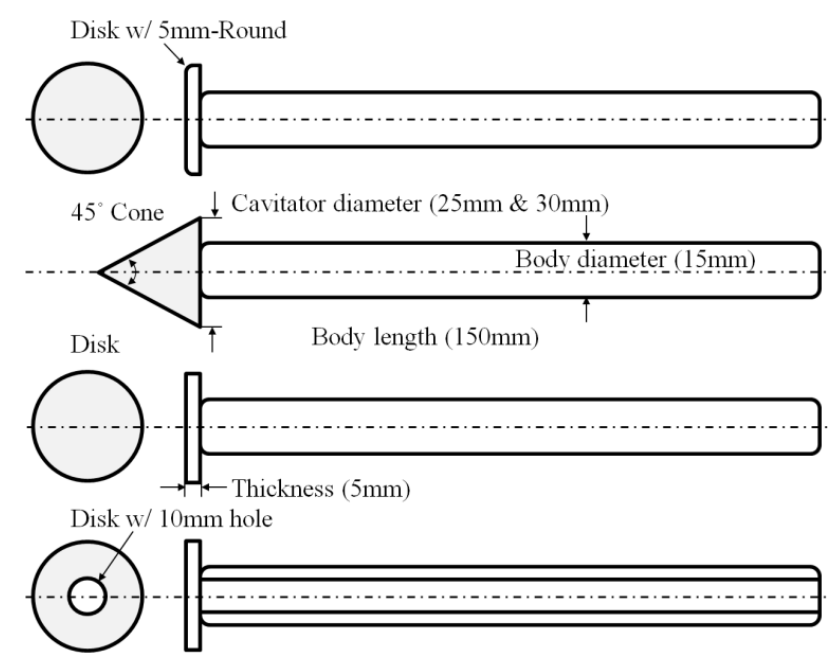

Fig. 11 Configurations of three-dimensional cavitators.

Measured cavity length and maximum width for the models are shown in Fig. 12 and Fig. 13 respectively. These cavity shapes are non-dimensionalised by the cavitator diameter. All data show consistent variation with cavitation number; as cavitation number is decreased, in other words, as flow speed increased, cavity length and width are increased. As expected, disk shape cavitator generates longer and thicker cavities comparing with cone and rounded plate shape cavitators. There is little scale effect in case of the disk, but it appears in both cases of cones and rounded tip disks. In this try, measured data were resulted from film strips recorded by using a general video camera. As explained in previous chapter, one may overestimate cavity dimensions obtained from low frame records. It is believed that these discrepancies were resulted from this reason, and blockage effect was also involved. It is, however, sufficient to find general features of the supercavity according to each stage of the reduced cavitation number. Recorded film strips of supercavitation at certain cavitation numbers after cavity inception are shown in Fig. 14.

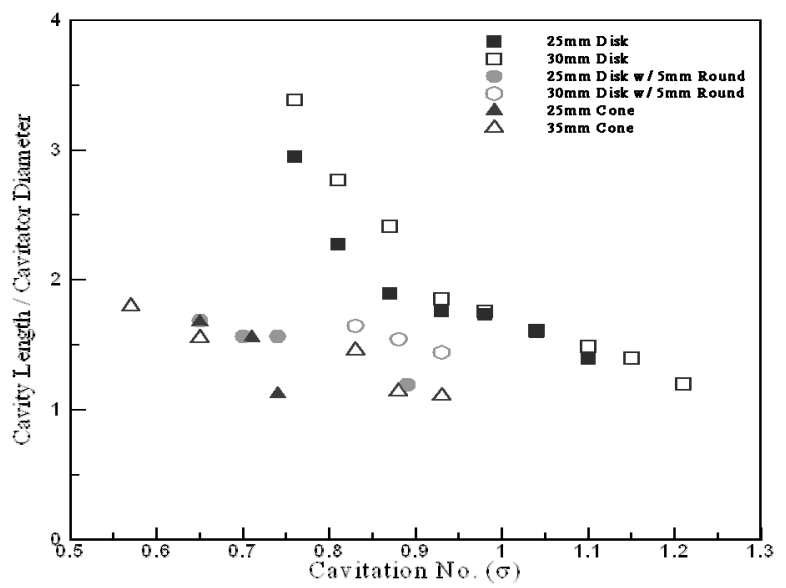

Fig. 12 Non-dimensional cavity length versus cavitation number for different three-dimensional cavitators.

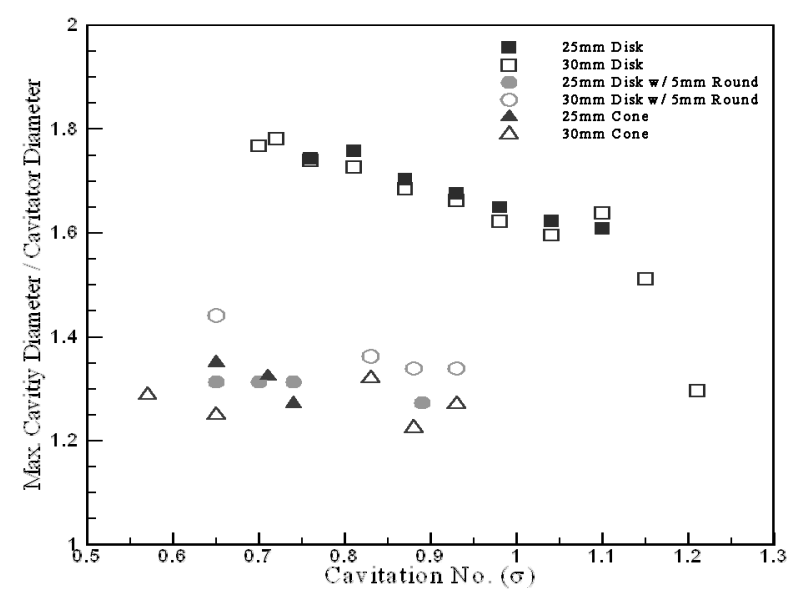

Fig. 13 Non-dimensional cavity width versus cavitation number for different three-dimensional cavitators. 
The detachment point is located clearly at the edge of the cavitator in all cases. At the low cavitation number, the cavity pulsates periodically in length direction causing fluctuating forces on the cavitator, and interfaces between the cavity and the liquid are opaque and rough. As the cavity grows further and it reaches to the full-length supercavity, it becomes transparent and stable. Fig. 14 (a) and (b) show that, disk shape cavitators forms supercavity earlier than the cone shape cavitator. As discussed before, blunt cavitator generates much longer and wider cavity than the slender one at the same cavitation number. Fig. 14 (c) and (d) show the sequence of cavity growth with and without water intake. To varying degrees depending on the intake size, cavity growth is delayed in case of water passing at the center of the cavitator through the body. Oh the other words, full-length transparent supercavity is generated at lower cavitation number, and its width is little smaller than that of the cavitator without hole.

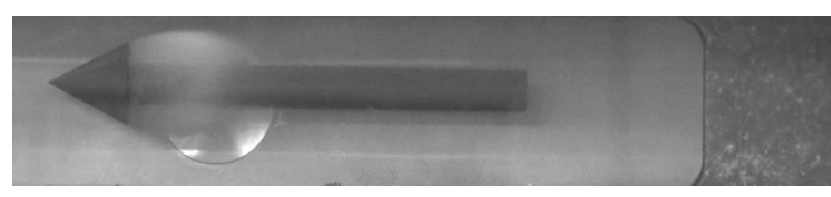

$\sigma=0.93$

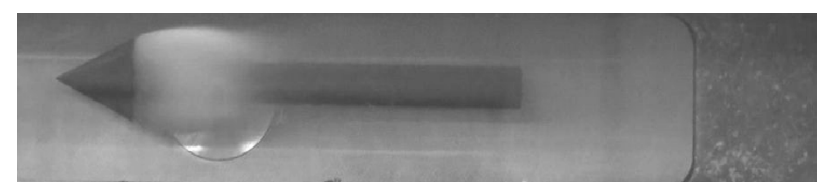

$\sigma=0.83$

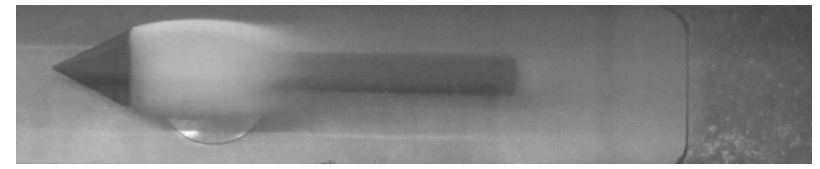

$\sigma=0.65$

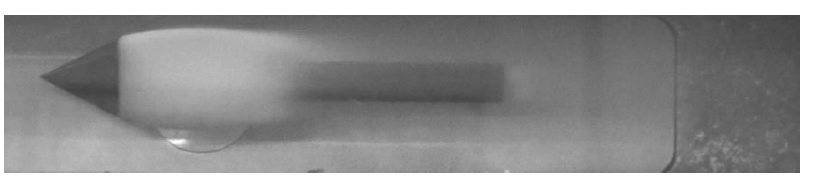

$\sigma=0.57$

(a) $45^{\circ}$ Cone.

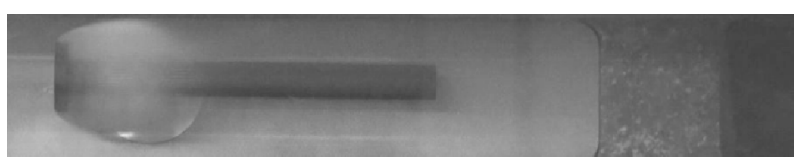

$\sigma=1.27$

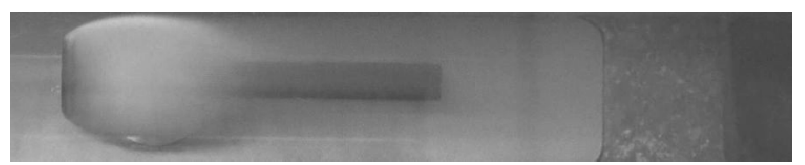

$\sigma=0.98$

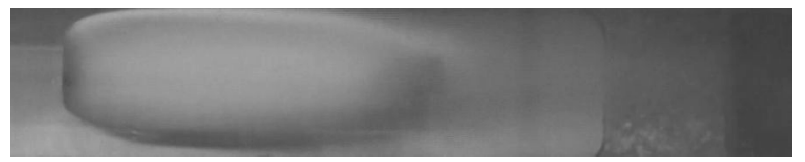

$\sigma=0.88$

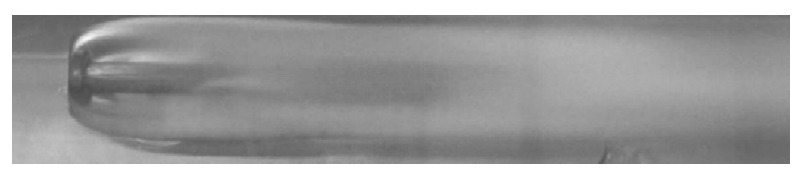

$\sigma=0.83$

(c) Disk without hole.

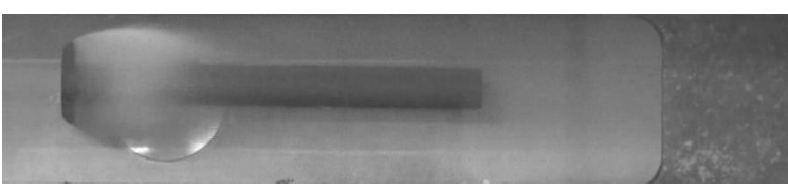

$\sigma=0.93$

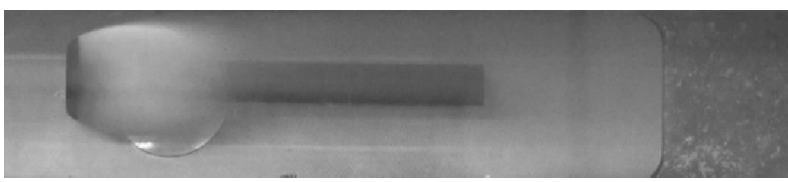

$\sigma=0.83$

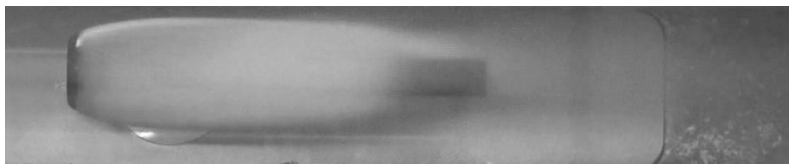

$\sigma=0.65$

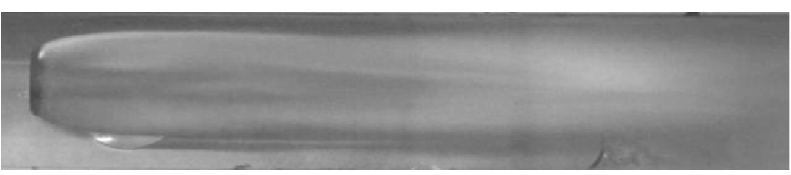

$\sigma=0.57$

(b) Disk with $5 \mathrm{~mm}$ round.

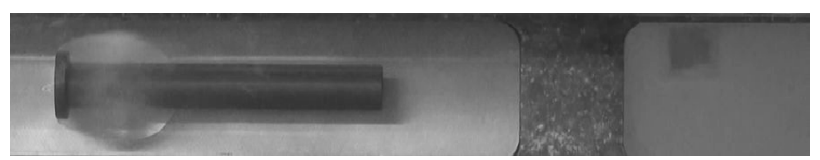

$\sigma=1.28$

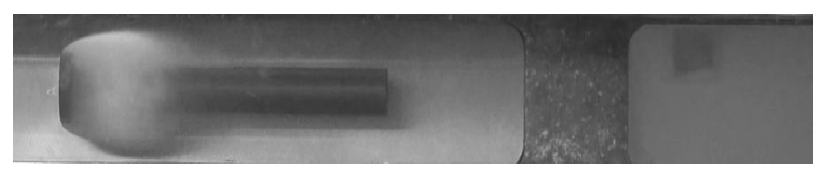

$\sigma=1.0$

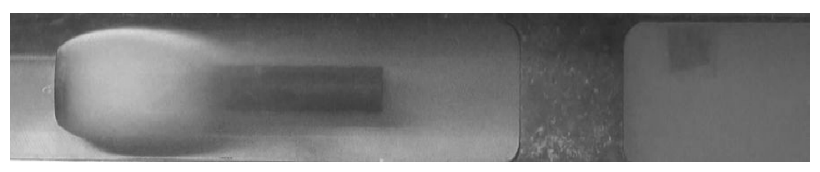

$\sigma=0.89$

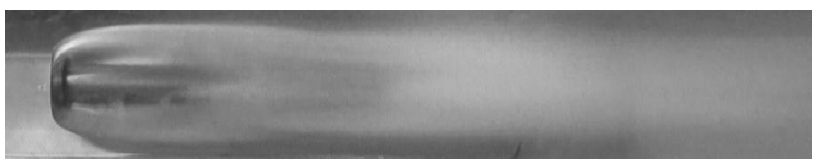

$\sigma=0.79$

(d) Disk with hole.

Fig. 14 Sequence of events of the supercavity in the wake of three-dimensional cavitators (flow speed $V=11.0 \mathrm{~m} / \mathrm{s}$ ). 


\section{SUMMARY AND CONCLUSIONS}

In the present work, we examined, first, general features of supercavitation by analyzing results obtained using previously developed numerical method; how cavity length and width are changed, and also how drag is reduced according to the cavitation number. Predicted results are validated by comparing with existing analytic solutions, and several cases of the twodimensional wedge type cavitator were investigated. Second, we observed supercavity profiles following wedge angle $30^{\circ}, 45^{\circ}$, and flat plates, and also measured the effects of the body placed behind the wedge and flat plate. In this try, we carefully examined the early stage of the supercavity using a high-speed camera, and showed fluctuating unsteadiness and its effect on supercavity dimensions.

Finally, we observed supercavity profiles generated by three-dimensional cone and disk shape cavitators. Results showed consistent features with two-dimensional cases. We also investigated that water intake from the center of the cavitator delays the growth of the cavity and decreases supercavity dimensions.

\section{ACKNOWLEDGEMENTS}

This study was financially supported by research fund of Chungnam National University in 2010.

\section{REFERENCES}

Ahn, B.-K., Lee, C.-S. and Kim, H.-T., 2010. Experimental and numerical studies on super-cavitating flow of axisymmetric cavitator. International Journal of Naval Architecture and Ocean Engineering, 2(1), pp.39-44.

Alyanak, E., Venkayya, V., Grandhi, R. and Penmetsa, R., 2004. Variable shape cavitator design for a supercavitating torpedo. Proc. of $10^{\text {th }}$ AIAA/ISSMO Multidisciplinary Analysis and Optimization Conference. Albany, NY, USA.

Gilbarg, D., 1957. Free stream theory and steady-state cavitation. Proc. Symposium On Naval Hydrodynamics. Washington, D.C., pp. 281-295.

Knapp, R.T., Daily, J.W. and Hammitt, F.G., 1979. Cavitation, McGraw-Hill.

Kunz, R.F., Lindau, J.W., Billet, M.L. and Stinegring, D.R., 2001. Multiphase computational fluid dynamics modeling of developed and supercavitating flows. Von Karman Institute for Fluid Dynamics, Rhode Saint Genese, Belgium.

Newman, J.N., 1977. Marine Hydrodynamics. Cambridge, MA, USA : The MIT Press.

Reichardt, H., 1946. The laws of cavitation bubbles at axially symmetric bodies in a flow. Ministry of Aircraft Production (Britain), Rep. and Transl. 766.

Saurel, R. and Le Metayer, O., 2001. A multiphase model for compressible flows with interfaces, shocks, detonation waves and cavitation. Journal of Fluid Mechanics, 431, pp.239-271.

Self, M. and Ripken, J.F., 1955. Steady-state cavity studies in a free-jet water tunnel. St. Anthony Falls Hydr. Lab. Rep. 47. Semenenko, V.N., 2001. Artificial Supercavitation, Physics and Calculation, Lecture Notes for the RTO AVT/VKI Special Course on Supercavitating Flows. Von Karman Institute for Fluid Dynamics, Rhode Saint Genese, Belgium.

Tulin, M.P., 1953. Steady two-dimensional cavity flows about slender bodies. DTMB Rep. 834.

Waid, R.L., 1957. Water tunnel investigation of two-dimensional cavities. CalTech. Hydrodyn. Lab. Rep. E-73.4. 